\title{
Delocalized States in Damaged DNA
}

\author{
R. A. Caetano and P. A. Schulz \\ Instituto de Física Gleb Wataghin, UNICAMP, Cx. P. 6165, 13083-970, Campinas, SP, Brazil
}

Received on 4 April, 2005

\begin{abstract}
Recent studies suggest that base pairing is an efficient electronic delocalization mechanism. However, defects may break down such effect. In the present work we show how a simple model of defects suppresses the delocalization, which survives only for low defect concentrations.
\end{abstract}

Keywords: Damaged DNA; Delocalization; Tight binding model

\section{INTRODUCTION}

The possibility of charge transference in DNA molecules along stacked $\pi$ orbitals was proposed by Eley and Spivey 40 year ago [1]. Since this work, the interest in its electronic properties has been growing, specially in the last decade. The particular binding between single strands of DNA, the controled growth of DNA molecules and its self-assembly properties have pointed to DNA molecules as a promissor molecular electronic component [2]. Many efforts have a biochemical motivation, since sensing damage mechanism may explore the long range electron migration along the molecule [3]. However, the main question about this topic is still unsolved. Experimental works on DNA conductivity are very controversial: Metallic [4], semiconductor [5], insulating [6] and even superconcuctor [7] behaviors have been reported. These experiments are very complex due to the local enviroment, such as counterions, contact resistence, thermal vibrations and even sequence variability, wich are difficult to control in non designed samples [8]. However, it has been shown that deliberately induced damage to DNA molecules can significantly reduce electron migration [9].

Theoretical works range from a strictly one dimensional tight binding $[10,11]$ up to involved $a b$ initio and density functional methods $[8,12]$. Both approaches have partial success, but with severe limitations. Strictly one dimentional tight binding models deal with sigle chains, so that the base pairs correlations are not taken into account. On the other hand, $a b$ initio calculations have a limited number of DNA molecule models. A recent study models the DNA molecules as a double chain. In this way, the base pairs correlation is properly taken into account and reveals itself as efficient delocalization mechanism[13].

In this work, we extend a previous study [13] to show that low concentration damages induced in DNA molecules do not destroy completely the delocalized states, but increasing the deffects concentration, just localized states survive.

We use a double-chain nearest-neighbor tight binding model to describe the system. In this approximation, the Hamiltonian can be written as:

$$
H=\sum_{i=1}^{N / 2} \sum_{j=1}^{2}\left[\varepsilon_{i, j}|i, j\rangle\langle i, j|+V| i+1, j\rangle\langle i, j|+V| i-1, j\rangle\langle i, j|\right.
$$

$$
\left.+V^{\prime}|i, j+1\rangle\left\langle i, j\left|\delta_{j, 1}+V^{\prime}\right| i, j-1\right\rangle\langle i, j| \delta_{j, 2}\right]
$$

where $\varepsilon_{i, j}$ is the $(i, j)$ site energy, $V$ is the intra-chain hopping parameter, $V^{\prime}$ is the inter-chain hopping parameter and $N / 2$ is the number of base pairs ( $N$ is the total number of sites).

We represent the four nucleotides which compose the double chain by $\mathrm{A}, \mathrm{T}, \mathrm{C}$ and $\mathrm{G}$ in fig 1 . In the first chain these nucleotides are randomly assingned with equal concentration on average. The sites of the second chain obey the base pairing.

DNA molecules are frequently damaged by oxidation due to intracellular chemistry or extracellular ionization radiation [2]. Here, defects are represented by inter-chain dangling bonds, e.g., $V^{\prime}=0$.

Figure 1 shows a particular damaged DNA configuration. Spurious effects due to a special configurations are avoided by taking an average over 20 DNA configurations.

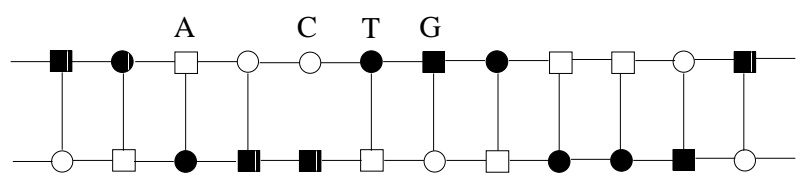

FIG. 1. Particular configuration of damaged DNA molecule .

In order to decide wheter or not a state is delocalized, we used the Participation Ratio [14], defined, in tight binding approximation, by:

$$
P R=\frac{1}{N \sum_{i=1}^{N / 2} \sum_{j=1}^{2}\left|a_{i, j}\right|^{4}}
$$

where $a_{i j}$ is the normalized wave function amplitude in the $(i, j)$ site. PR is close to zero for localized states for $N \rightarrow \infty$ and for a delocalized states it reaches the maximal value of $2 / 3$ in a one dimensional system [15], if cosine like modulation of Bloch states are taken into account.

\section{RESULTS}

In order to investigate the influence of the damages on DNA molecules, we use a previously tested tight binding 
parametrization. The site energies are [16]: $\varepsilon_{A}=8.24 \mathrm{eV}$, $\varepsilon_{T}=9.14 \mathrm{eV}, \varepsilon_{C}=8.87 \mathrm{eV}$ and $\varepsilon_{G}=7.75 \mathrm{eV}$. Hopping parameters describes the $\pi-\pi$ orbitals interactions between base pairs. Ab initio calculations found that hopping parameter is of the order of $0.44 \mathrm{eV} \mathrm{[16].} \mathrm{However,} \mathrm{a} \mathrm{previous}$ work considered DNA molecules conected to contacts with a hopping parameter equal to $1 \mathrm{eV}$, motivating our choice $V=1 \mathrm{eV}$ [16]. Preceding works have suggested that inter chain hopping parameter $V^{\prime}$ is smaller than intra chain hopping parameter $V[6,17]$. In this work we use $V^{\prime}=0.5$.

Figure 2 and Fig. 3 compare the participation ratio of a perfect (without damages) DNA molecules (dashed line in Fig. 2) with one that has five per cent of damaged bindings (Fig. 3). In Fig. 2 we also show the participation ratio of a DNA that do not present base pairing (continuos line).

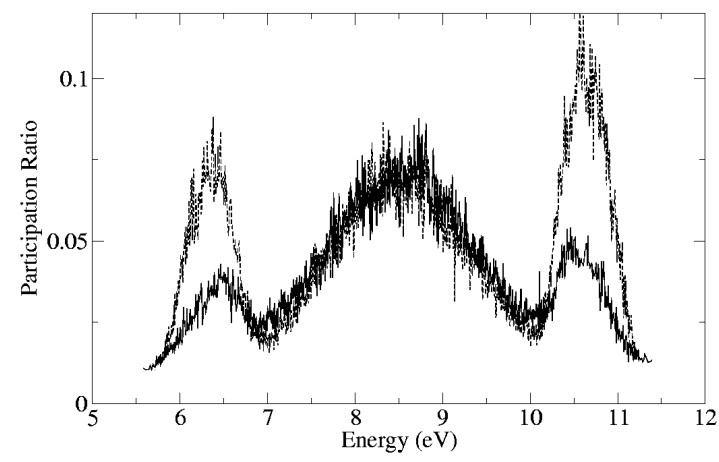

FIG. 2. Participation ratio of 500 base pairs DNA molecules for: Perfect DNA molecule (dashed line) and a non base pairing DNA (continous line). The tight binding parameters are given in the text.

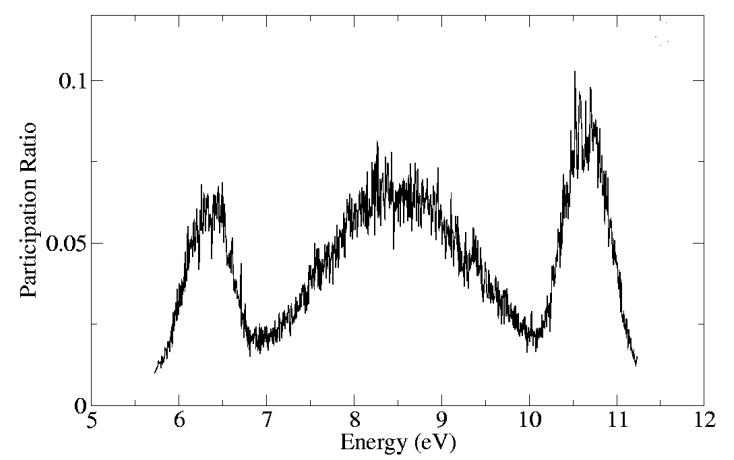

FIG. 3. Participation ratio of 500 base pairs DNA molecules for a damaged DNA with 5 per cente of damaged binding.

Although Figs. 2 and 3 show a clear decrease of the participation ratio, with increasing number of dangling bonds, delocalized states still continue to be present. In Fig. 4 we show a delocalized state, with energy near $10.5 \mathrm{eV}$, for a 5 per cent concentration of dangling bonds. It is worthy of mentioning that the wave function spread out along the entire chain.

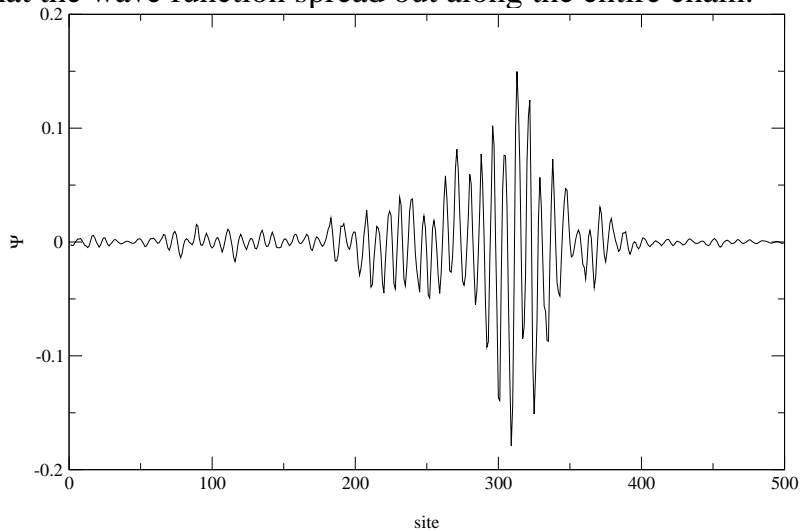

FIG. 4. Wave function of a delocalized state with energy close to $10.5 \mathrm{eV}$.

Increasing the damage concentration, the delocalized states are washed out. The evolution of the maximal value of the participation ratio with increasing the defect concentration is shown in Fig. 5.

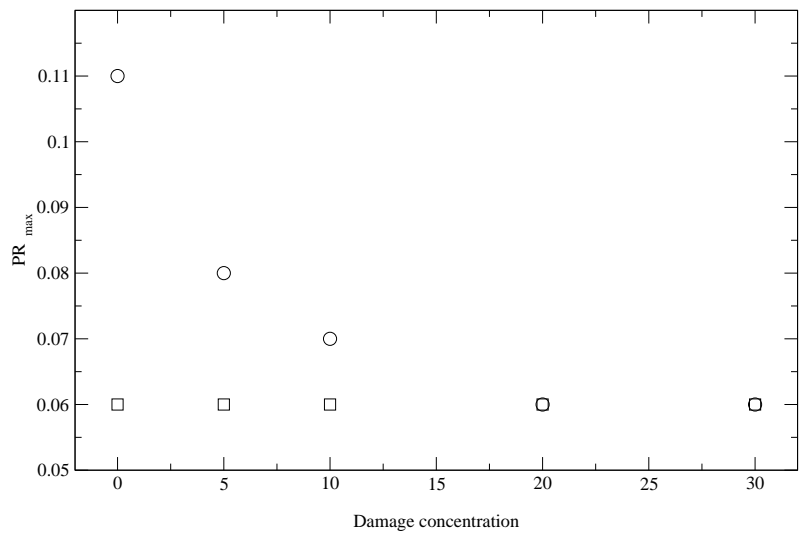

FIG. 5. Maximal value of participation ratio as function of damage concentration (circle). maximal participation ratio of a no base pairing (square).

Figure 5 shows that above 20 per cent of damaged bonds concentration, base pairing does not work as delocalizaton mechanism and all states are localized.

\section{CONCLUSION}

In conclusion, we study damages in DNA molecules. In a simple model, we show that low damage concentration does not suppress all delocalized states. Increasing the damage concentration, only localized states remain and the base pairing does not work as a delocalizaton mechanism anymore, and in this way DNA behaves like an insulator. 
[1] D. D. Eley and D. I. Spivey, Trans. Faraday Soc. 58, 411 (1961).

[2] R. G. Endres, D. L. Cox, and R. P. Singh, Rev. Mod. Phys. 76, (2004).

[3] P. J. Dandliker, R. E. Holmlin, and J. K. Barton, Science 275 , 1465-1468 (1997).

[4] Hans-Werner Fink and Christian Schönenberg, Nature, 398, 407-410 (1999).

[5] Danny Porath, Alexey Bezryadin, Simon de Vries, and Cees Dekker, Nature, 403 (2000).

[6] P. J. de Pablo, F. Moreno-Herrero, J. Colchero, J. Gómez Herrero, P. Herrero, A. M. Baró, Pablo Ordejón, José M. Soler, and Emilio Artacho, Phys. Rev. Lett. 85, 492-495 (2000).

[7] A. Kasumov, M. Kociak, S. Guéron, B. Reulet, V. Volkov, D. Klinov, and H. Bouchiat, Science 275, 280, (2001).

[8] Haw Wang, James P. Lewis, and Otto F. Sankey, Phys. Rev. Lett. 93, 016401-1 (2004).
[9] S. O. Kelley, N. M. Jackson, M. G. Hill, and J. K. Barton, Angew. Chem., Int. Ed. Engl. 38, 941, (1999).

[10] P. Carpena, P. Bernaola-Galvan, P. Ch. Inavov, and H. E. Stanley, Nature 418, 955 (2002)

[11] Wei Zhang and Sergio Ulloa, Phys. Rev. B, 69, 153203, (2004).

[12] Ch. Adessi S. Walch and M. P. Anantram, Phys. Rev. B, 67, 081408(R), (2003)

[13] R. A. Caetano and P. A. Schulz, Phys. Rev. Lett., 95, 126601, (2005).

[14] Dean P. and Bell R., Discuss. Faraday Soc., 50, 55-61, (1970).

[15] D. J. Thouless, Phys. Report, 13, 93, (1974).

[16] S. Roche and E. Maciá, Moder. Phys. Lett. B, 18, 17, (2004).

[17] P. Maragakis, R. L. Barnett, E. Kaxiras, M. Elstner, and T. Frauenheim, Phys. Rev. B, 66, 241104(R) (2002). 